\title{
CLOSED GEODESICS ON RIEMANN SURFACES
}

\author{
TROELS JØRGENSEN ${ }^{1}$
}

\begin{abstract}
This note deals with an elementary property of closed geodesics on surfaces with a complete metric of constant negative curvature.
\end{abstract}

\section{Introduction.}

THEOREM. Any point which is the proper intersection of two (not necessarily different) closed geodesics is a point of intersection of infinitely many different closed geodesics.

This property, though quite easy to prove, seems not to have been observed before. We will show how it is revealed from hyperbolic space geometry.

2. Hyperbolic space. The upper half-space $\mathbf{H}=\mathbf{C} \times \mathbf{R}_{+}$equipped with the metric $d s^{2}=t^{-2}\left(|d z|^{2}+d t^{2}\right)$ provides us with a model of the hyperbolic space. In this model, which was introduced by Poincaré, the hyperbolic straight lines are euclidean half-circles and half-lines perpendicular to the complex plane.

The orientation-preserving isometries of $\mathbf{H}$ are complex Möbius transformations $A(h)=(a h+b)(c h+d)^{-1}$, ad $-b c=1$, where the points $h=z+$ $t j$ of the space are thought of as a subset of the quaternion algebra. The group of all such isometries may be identified with $\operatorname{PSL}(2, \mathbf{C})$, that is, the multiplicative group of pairs of opposite complex unimodular 2-by-2 matrices.

The action of these isometries extends naturally to "the points at infinity", $\mathbf{C} \cup\{\infty\}$. Thereby, the usual action of Möbius transformations on the Riemann sphere is regained.

3. Invariant lines. Every elliptic or loxodromic Möbius transformation has a unique invariant hyperbolic line. This line is called its axis. It is of course the hyperbolic line determined by the two fixed points on the Riemann sphere.

A parabolic transformation does not have any invariant line. This is clear, since a parabolic transformation has only one fixed point. However, it is sometimes convenient to speak of this fixed point as "the axis".

4. Lie-products. Let $A$ and $B$ denote two elements of $\operatorname{SL}(2, \mathrm{C})$. Their Lie-product $A B-B A$ has determinant equal to $2-\tau\left(A B A^{-1} B^{-1}\right)$, where $\tau$ is the trace of a matrix. This determinant is different from 0 if and only if the Möbius transformations (represented by) $A$ and $B$ do not have any common

Received by the editors October 24, 1977 and, in revised form, February 15, 1978.

AMS (MOS) subject classifications (1970). Primary 53A35.

${ }^{1}$ Supported in part by the National Science Foundation. 
fixed points on the Riemann sphere. When this is the case, the Lie-product of $A$ and $B$ determines a Möbius transformation $\varphi$ which is elliptic of order 2 (its trace being 0 ).

It is true for any element $X$ of $\operatorname{SL}(2, \mathrm{C})$ that

$$
X+X^{-1}=\tau(X) E,
$$

where $E$ denotes the unit matrix. With use of this, it follows that

$$
(A B-B A)-\left(B A^{-1}-A^{-1} B\right)=\left(A+A^{-1}\right) B-B\left(A+A^{-1}\right)=0
$$

and

$$
(A B-B A)-\left(B^{-1} A-A B^{-1}\right)=A\left(B+B^{-1}\right)-\left(B+B^{-1}\right) A=0,
$$

which, together with the identities

$$
(A B-B A) A^{-1}=A\left(B A^{-1}-A^{-1} B\right)
$$

and

$$
(A B-B A) B^{-1}=B\left(B^{-1} A-A B^{-1}\right),
$$

shows that

$$
A=\varphi A^{-1} \varphi^{-1} \text { and } B=\varphi B^{-1} \varphi^{-1} .
$$

It follows that the mapping $\varphi$ interchanges the fixed points of $A$ as well as the fixed points of $B$. The geometrical content of this is that the axis of $\varphi$ is the common perpendicular of the axis of $A$ and the axis of $B$, suitably interpreted in case of $A$ or $B$ being parabolic.

5. Symmetry. The axes of those isometries, different from the identity, which are symmetric products of $A$ and $B$ must all be perpendicular to the axis of $\varphi$, because any such element $g$ satisfies

$$
g=\varphi g^{-1} \varphi^{-1}
$$

and, hence, $\varphi$ interchanges its fixed points.

6. Hyperbolic planes. In the Poincare model, the hyperbolic planes are euclidean hemispheres and half-planes perpendicular to the complex plane. The axis of an isometry preserving a plane either belongs to that plane or is perpendicular to it. The latter occurs only for elliptic transformations. Two intersecting hyperbolic lines belong to a unique hyperbolic plane. Their common perpendicular line meets that plane orthogonally (in one point). Hence, if two such lines are the axes of two transformations $A$ and $B$ preserving the plane, then the axes of all the symmetric elements with respect to $A$ and $B$ belong to the same plane and pass through a common point.

REMARK. It is easy to show that if the axes of $A, B$ and $A B A$ are oriented towards the attractive fixed points, then the axis of $A B A$ points into the first quadrant with respect to the axes of $A$ and $B$. It follows that the elements $A^{n} B A^{n}, n \in \mathrm{N}$, have different axes (which accumulate at the axis of $A$ ). 
7. Closed geodesics. Consider a surface with a complete metric of constant curvature equal to -1 . Its universal covering space is the hyperbolic plane. The covering transformations may be regarded as isometries of $\mathbf{H}$, preserving a plane on which they act freely and discontinuously. When lifted from the surface to this plane, the closed geodesics extend to (conjugacy classes of) axes of the respective covering transformations. If two such axes intersect, then the infinitely many axes passing through the same point must project onto an infinite number of different closed geodesics (but not necessarily one to one). Otherwise, two of these projections would intersect each other (or one would intersect itself) an infinite number of times, which is impossible by compactness and local properties of the geodesics.

Department of Mathematics, University of Minnesota, Minneapolis, Minnesota 55455 